\title{
Quantitative assessment of the masseter muscle's elasticity using Acoustic Radiation Force Impulse
}

\author{
Iulia Badea ${ }^{1 *}$, Attila Tamas-Szora ${ }^{2 *}$, Ioana Chiorean ${ }^{3}$, Floarea Fildan ${ }^{4}$, Elisabeta Ciuleanu ${ }^{5}$, \\ Mîndra Badea ${ }^{1}$
}

\begin{abstract}
${ }^{1}$ Department of Preventive Dentistry, "Iuliu Haţieganu" University of Medicine and Pharmacy, ${ }^{2}$ Imaging Department, "O. Fodor" Institute of Gastroenterology and Hepatology, "Iuliu Haţieganu” University of Medicine and Pharmacy, ${ }^{3}$ Faculty of Mathematics and Informatics, Department of Mathematics, Babes-Bolyai University, ${ }^{4}$ Department of Maxilo-Facial Radiology, "Iuliu Haţieganu" University of Medicine and Pharmacy, ${ }^{5}$ Department of Radiotherapy, Oncologic Institute "Prof.dr. Ion Chiricuţă" Cluj Napoca, Romania

* the authors shared the first authorship
\end{abstract}

\begin{abstract}
Aims: The study proposes Acoustic Radiation Force Impulse (ARFI) assessment of the masseter muscle elasticity in the healthy population and in patients who have undergone head and neck radiation therapy. Patients and methods: Twenty-five healthy controls constituted group A, and 13 patients who had underwent radiotherapy (35Gy minimum) formed group B. ARFI was performed bilaterally in the periphery $(\mathrm{P})$ and the muscle center $(\mathrm{C})$, in relaxation and contraction. Means and standard deviations were obtained for the recorded shear waves velocities (SWV). Results: For group A: in the relaxed right muscle $\mathrm{C}$ $=1.87 \pm 0.52 \mathrm{~m} / \mathrm{s}$ and $\mathrm{P}=1.96 \pm 0.55 \mathrm{~m} / \mathrm{s}$ and in the left muscle $\mathrm{C}=1.66 \pm 0.47 \mathrm{~m} / \mathrm{s}, \mathrm{P}=1.67 \pm 0.53 \mathrm{~m} / \mathrm{s}$. For group $\mathrm{B}$, in relaxation, the right side presented $\mathrm{C}=1.67 \pm 0.6 \mathrm{~m} / \mathrm{s}, \mathrm{P} 1.72 \pm 0.56 \mathrm{~m} / \mathrm{s}$, the left muscle $\mathrm{C}=1.6 \pm 0.34 \mathrm{~m} / \mathrm{s}, \mathrm{P}=1.73 \pm 0.37 \mathrm{~m} / \mathrm{s}$. There were no differences $(\mathrm{p}>0.05)$ between $\mathrm{P}$ and $\mathrm{C}$ of both groups, regardless of the muscle state. The values for relaxation and contraction in $\mathrm{A}$ and $\mathrm{B}$ (merged values of the right and left) presented no differences $(1.79 \pm 0.52 \mathrm{~m} / \mathrm{s}$ vs $1.72 \pm 0.73 \mathrm{~m} / \mathrm{s}, \mathrm{p}=0.72$ and $1.70 \pm 0.48 \mathrm{~m} / \mathrm{s}$ and vs $1.59 \pm 0.77 \mathrm{~m} / \mathrm{s}, \mathrm{p}=0.98)$. All measurements of group A vs B were not different $(1.79 \pm 0.52 \mathrm{~m} / \mathrm{s}$ vs $1.65 \pm 0.63 \mathrm{~m} / \mathrm{s}, \mathrm{p}=0.78)$. Conclusions: ARFI with SWV measurement enables the quantification of normal and post irradiation elasticity of the masseter. Further studies on a larger population are required to validate the normal and pathologic values.

Keywords: ARFI, masseter muscle, radiation, relaxation, contraction
\end{abstract}

\section{Introduction}

The elasticity of tissues is influenced by particular physiological as well as pathological entities. In the masseter muscle the most common pathological conditions

Received 10.02.2014 Accepted 30.03.2014

Med Ultrason

2014, Vol. 16, No 2, 89-94

Corresponding author: Attila Tamas-Szora

Imaging Department, "O. Fodor" Gastroenterology and Hepatology Institute

"Iuliu Hațieganu" University of Medicine and Pharmacy

19-21 Croitorilor str,

400162 Cluj Napoca, Romania

Phone: +40740084968

E-mail: tamas-szora.attila@umfcluj.ro encountered are hypertrophy, myositis, and rhabdomyosarcoma. Frequently the muscle is affected in some neurological syndromes (paresis, paralysis), and, as in the case of older patients, it becomes atrophic [1]. It is known that tooth extraction and the subsequent decrease in the number of functional tooth units, in the advanced stages, can lead to decreased masticatory muscle force. Some authors have described that changes in muscle thickness can be recovered after oral rehabilitation [2]. Various entities such as occlusal disharmony, disruption of the dynamics in the temporomandibular joint (TMJ), and bruxism reflect on the masseter, causing stress, tension, and pain. Moreover, the involvement of the masseter muscle in the myofascial pain syndrome is recognized, and this pathology can evolve with hemifacial swelling $[3,4]$. 
Sonoelastography is a relatively new technique that allows the investigation of tissue elasticity. The two directions in the development of the method are based on the evaluation of the strain stress and of the shear waves. Both are non-invasive, but the determination of the propagation velocity of the shear waves (SWV) using the Acoustic Radiation Force Impulse method (ARFI) holds the advantages of a quantitative estimation and a reduced operator dependency.

There are numerous published studies concerning the diagnostic benefit of the method in different situations. Special interest was given to the assessment of liver pathology $[5,6]$. In terms of applications on the neck and maxillofacial region, the focus was directed mainly on the oncological pathologies involving the lymph nodes [7-9], thyroid [10,11] and salivary glands [12-14]

Studies regarding the ARFI evaluation of the masticatory muscles are scarce. From the available data, this study is the first to question the feasibility of the ARFI method on the masseter muscle. The study proposed two principal objectives: firstly, the evaluation of the masseter muscle stiffness in the general population, taking into account the implications associated with the type of mastication and, secondly, the evaluation of the muscle stiffness secondary to radiotherapy in the head and neck region.

\section{Material and methods}

This prospective study was conducted during February-April 2013 and received the approval from the Ethics Committee of the University of Medicine and Pharmacy Cluj-Napoca. Prior to admission the patients were informed of its purpose and an informed consent was obtained from all participants.

The study group included 25 voluntary healthy pa-

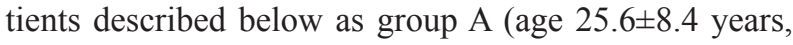
$M: F=9: 16$ ). The inclusion criteria were: unaltered general state, absence of maxillofacial trauma, absence of maxillofacial surgery, absence of myofascial symptoms, normal occlusal status, and no history of TMJ alterations.

Thirteen patients who underwent radiotherapy for nasopharyngeal carcinoma were allotted to group B (age $50 \pm 11.93$ years, $\mathrm{M}: \mathrm{F}=9: 4)$. The inclusion criteria were: a minimum dose of $35 \mathrm{~Gy}$, a minimum interval of six months after the last exposure to radiation, the absence of surgery in the masseter region, good health status. During radiation therapy planning, all of the critical adjacent structures to the nasopharynx (e.g. brainstem, optic lens, salivary glands) including the masseter, were carefully outlined and the doses to these organs were lowered as much as possible, without affecting coverage of the tumoral target.

Before the ultrasound examination each patient was clinically examined and questioned about the masticatory predominance (classified as right, left, alternative or unknown).

Two experienced examiners performed the ultrasound examinations (ATS, IB). The equipment consisted of the Siemens S2000 equipped with an 8-14 MHz linear transducer and the software for the ARFI quantitative examination.

Patients were positioned on the examination bed in a supine position with the head half extended. The muscle assessment was performed bilaterally in relaxation and contraction. In the case of muscle contraction, the patients were asked to perform a moderate contraction. In both cases patients were asked to suppress swallowing.

The grey scale examination was performed at a frequency of $8 \mathrm{MHz}$, using a single focus located under the muscle. The section planes were positioned perpendicular and parallel to the direction of the muscle fibres, consequently measuring the maximum thickness of the muscle in the middle third of the ramus of the mandible.

Colour Doppler ultrasound examination was used to identify the main intramuscular vascular branches.

Consequently the ARFI mode was activated. The predefined region of interest (ROI) was placed just below the superficial fascia - described here as the periphery, and at the center of the muscle immediately below the peripheral region - hereinafter referred to as the center (fig 1). The main vascular branches and the intramuscular fascial components were avoided. The focus was placed on the region of interest. In order to avoid possible errors, the pressure applied by the transducer on the skin was maintained at a minimum level [15].

The optimal assessment was characterized by obtaining 10 valid measurements of the area of interest, regardless of the number of repetitions needed. Invalid measurements were represented by the system as XXXX / 0". The SWV values were expressed in $\mathrm{m} / \mathrm{s}$.

The statistical analysis was performed using SPSS Statistics (version 20) and Microsoft Excel 2007. For the comparison of means the Pearson test was used. The same test was applied to identify correlations (sex and the type of chewing in relation to the SWV values). The level of statistical significance was set at $p=0.05$. Data are presented as mean \pm standard deviation $(\mathrm{M} \pm \mathrm{SD})$.

\section{Results}

In group A for the relaxed muscle, there were no difficulties in achieving the required ARFI measurements in 


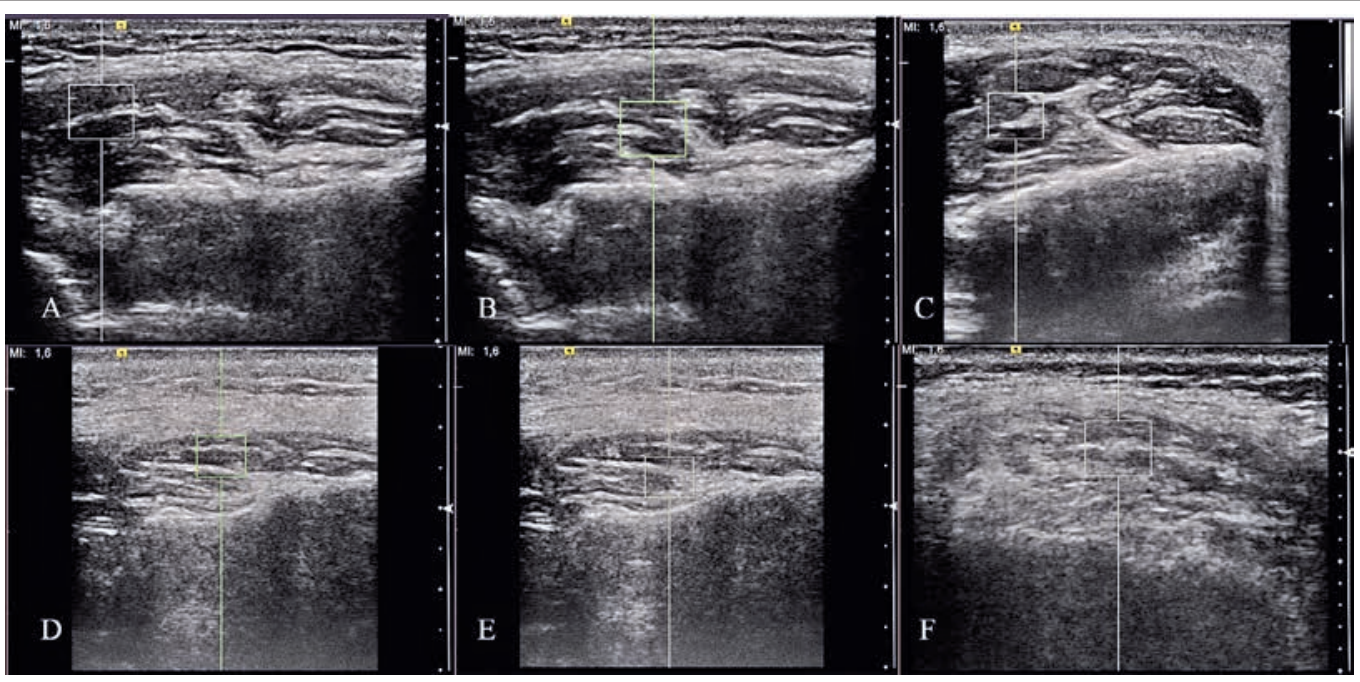

Fig 1. ARFI examination of a patient in group A: relaxation, periphery (A), relaxation, central (B), contraction, central (C). For one patient in group B: relaxation, periphery (D), relaxation, central (E), contraction periphery $(\mathrm{F})$.

both central and peripheral locations. For group B there were impediments in two patients at both the central and peripheral left masseter. The suboptimal measurements obtained were ignored in the final statistics.

The examination during contraction was insufficient for one healthy subject, in the right side (periphery and center). In group B, in 3 patients there were difficulties in obtaining the required number of values in the periphery of the right masseter. For these patients and another additional two patients, measuring the velocities in the masseter center was impossible as well. Six of the examinations on the left masseter muscle during contraction, peripherally, and 5 centrally were excluded.

For the measurements acquired in the optimal range, the SWV $(\mathrm{m} / \mathrm{s})$ values are presented in table I and II

When comparing between the periphery and the cent$\mathrm{er}$, the mean values of the measurements did not reach statistical significance (all $\mathrm{p}>0.05$ ) regardless of the group or location analysed.

When assessing the patients during contraction, although subjectively there were differences between the mean values obtained in the central and peripheral muscle, both in group A and B (in the right and the left muscle), there was a significant overlap of values, and the differences did not reach the statistical significance threshold $(p>0.05)$. Similarly, the concatenated mean values of the center and periphery for contraction and relaxation did not reach the level of statistical significance, when comparing the right to the left muscle within each group (table III).
Table I. Means and standard deviations of ARFI measurements $(\mathrm{m} / \mathrm{s})$ obtained for the masseter in relaxation.

\begin{tabular}{ccccc}
\hline & \multicolumn{2}{c}{ Right masseter } & \multicolumn{2}{c}{ Left masseter } \\
\hline & Central & Peripheral & Central & Peripheral \\
\hline Group A & $1.87 \pm 0.52$ & $1.96 \pm 0.55$ & $1.6 \pm 0.47$ & $1.67 \pm 0.53$ \\
Group B & $1.67 \pm 0.6$ & $1.72 \pm 0.56$ & $1.6 \pm 0.34$ & $1.73 \pm 0.37$ \\
\hline
\end{tabular}

Results are shown as mean \pm standard deviation

Table II. Means and standard deviations of ARFI (m/s) measurements obtained at the examination during contraction.

\begin{tabular}{ccccc}
\hline & \multicolumn{2}{c}{ Right masseter } & \multicolumn{2}{c}{ Left masseter } \\
\hline & Central & Peripheral & Central & Peripheral \\
\hline Group A & $1.57 \pm 0.54$ & $1.81 \pm 0.9$ & $1.47 \pm 0.53$ & $1.74 \pm 0.72$ \\
Group B & $1.41 \pm 0.52$ & $1.48 \pm 0.64$ & $1.68 \pm 0.9$ & $1.84 \pm 0.9$ \\
\hline
\end{tabular}

Results are shown as mean \pm standard deviation

Table III. ARFI means and standard deviations $(\mathrm{m} / \mathrm{s})$ from concatenated central and periphery in the groups' study.

\begin{tabular}{llcc}
\hline & Right masseter & Left masseter \\
\hline Group A & relaxation & $1.86 \pm 0.52$ & $1.70 \pm 0.53$ \\
& contraction & $1.6 \pm 0.7$ & $1.86 \pm 0.76$ \\
Group B & relaxation & $1.70 \pm 0.57$ & $1.70 \pm 0.34$ \\
& contraction & $1.44 \pm 0.57$ & $1.76 \pm 0.94$ \\
\hline
\end{tabular}

Results are shown as mean \pm standard deviation 
Consequently no significance was identified when comparing the values recorded in relaxation and contraction for healthy patients (obtained by concatenating the values of the right and left muscle, $1.79 \pm 0.52 \mathrm{~m} / \mathrm{s}$ vs $1.72 \pm 0.73 \mathrm{~m} / \mathrm{s}, \mathrm{p}=0.72$ ). A similar result was noted in the case of group B (relaxation $1.70 \pm 0.48 \mathrm{~m} / \mathrm{s}$, contraction $1.59 \pm 0.77 \mathrm{~m} / \mathrm{s}, \mathrm{p}=0.98)$.

The comparative evaluation of the measured values in the relaxed state between the group A and B, and in the contracted state, did not find a statistically significant difference.

For the entire group A, the mean values of the measurements were $1.79 \pm 0.52 \mathrm{~m} / \mathrm{s}$, and for group B 1.65 $\pm 0.63 \mathrm{~m} / \mathrm{s}$. These findings had no disparity ( $\mathrm{p}=0.78$ ).

When testing the association, in group $\mathrm{A}$, between the ARFI values and respectively the type of mastication (right / left) and gender, no relevant correlations could be made.

\section{Discussions}

The masseter muscle has a complex internal structure. It's a "feather" muscle, with three insertions originated at the level of the zygomatic arch, which outline the three components of the muscle (superficial, intermediate and profound). Distally the muscle is inserting to the angle and ramus of the mandible [16]. The internal inhomogeneity visible during the ultrasound examination is due to internal aponeurosis [16] identified as echogenic bands.

The direct evaluation of the physical processes that occur during muscle contraction is difficult under normal functioning. One reason is because inhomogeneous contractions, which occur in various parts of a complex muscle, produce different internal forces and regional changes in intramuscular pressure [17]. In addition, the fibers of a muscle, which is attached at an end on a tendon and distally on a bone surface, can be shortened by different degrees and at different angles [18].

The main research regarding the ultrasound examination of the masticatory muscles and therefore the masseter consisted, until recently, of identifying the morphological changes, in B mode, associated with the spectrum of TMJ impairments $[19,20]$. The masticatory musculature is frequently affected in this pathological context, in terms of functional efficiency, electrical activity and force of contraction [21].

Recently, elastography was introduced as an examination method in the study of muscle physiology in normal and pathological conditions [22-26].

For the problem investigated in the present research the most relevant of these studies was conducted by Kemal et al [22]. The authors used the Supersonic Imaging
System to quantify the stiffness of the masseter muscle, and the values recorded were $10.4 \pm 3.7 \mathrm{kPa}$. The authors associated elevated elasticity with the male gender, without identifying a correlation between stiffness and age. However, compared to our study, the examination method does not describe the location of the muscle measurements and the number of values obtained / patient.

In the study conducted by Wen-Hsiu et al [27], the system used was a Siemens S2000 coupled with ARFI elastography and SWV evaluation. The structures examined were the following muscles: sternocleidomastoid, scalene, trapezius and levator scapula. Mean values and standard deviations were found in the range of $0.96 \pm-$ 0.09 (sternocleidomastoid) - $2.08 \pm 0.44$ (trapezius), and were significantly different among the muscles investigated. The study identified that values correlated with the presence of neck pain symptoms in the case of the trapezius. No significant correlations were found between SWV and gender, age, body mass index, left and right

Yoshiko et al described an interesting approach to the masseter muscle [28]. They used strain elastography (SE) and the Logiq E9 system (General Electric) in parallel with an MRI examination. They evaluated 10 healthy volunteers at rest after a muscular effort of 10 minutes and 10 minutes after the exercise. They concluded that after vigorous exercise muscle rigidity increases significantly.

The present research completes the above-mentioned studies and brings new information.

Although there is no evidence of the alternative damage to the various morphological components, both the superficial and deep structures of the muscle were evaluated. The importance of this lies in the assessment of the similarity of the two components in terms of elasticity. This observation has great practical application because the ARFI evaluation can thus be made in any of the muscle components, consequently reducing the need for numerous measurements. Finally this reduces the overall examination time.

However, the statement is relevant in the context of healthy patients, requiring further research, possibly in patients with TMJ pathology.

In addition, in agreement with the studies cited, we cannot identify a significant difference between the elasticity of the right and the left masseter muscles, in healthy or in post-irradiation subjects.

Muscle assessment was performed both at rest and during muscle contraction. The SWV values between the two states were similar. This study presents for the first time the SWV values for the masseter at rest, and can be regarded as a starting point for further research. One of the limitations of the study is that the contraction force 
was not evaluated objectively. Our results are important, however, even in this context, since there are no current studies evaluating the stiffness of the masseter muscle during contraction. Also, ARFI may develop into an independently standing method for selectively evaluating the contribution of the masseter and the temporalis muscle in the overall bite force. This may add to the quality standards of oral rehabilitation.

The lack of differences in the compared SWV between the two groups may be explained by two factors. One factor is revealed when reviewing the biological effects of radiation on the muscular tissue. The mature structures of human skeletal muscle show high resistance to radiation as morphological changes are concerned. In time necrosis may occur, following vascular and connective tissue disruptions [29]. Skin and muscle fibrosis is another side effect of radiotherapy. But its occurrence is late; some experimental studies using doses higher than those administered to patients in group B, described the development of muscle fibrosis at about five months after the radiation therapy [30]. Relating to the above, it is possible that the ARFI examination of group B occurred prior to the onset of morphological changes in the muscles. There is the alternative that the subtle muscle changes, in the early stage, are not recordable with ARFI. Another factor is that during radiation therapy planning and application, the temporal-manidibular joint, mandible, parotid and secondly the masseter were spared as much as possible. The overall ability to spare these structures was closely related to the extent and location of the nasopharyngeal tumor. The above mentioned factors represent limitations of our study, and require further evaluation. As the patients will be reevaluated one year after radiation therapy, these issues will be considered further. Also the lack of differences may be clarified if more evaluations are performed on patients receiving radiation therapy targeted more closely to the masseter region.

Given the differences in morphology between men and women, testing the correlation between the SWV and gender becomes important. Regarding the masseter, no statistically significant correlations were identified. Similarly there has not been identified a correlation between the SWV and the right or left masticatory predominance. We believe that this conclusion particularly requires validation by other studies on larger groups, because it may be a valuable criterion for assessing mastication imbalances in the future.

To summarize the above, we note that ARFI values in healthy and in particular postradiotherapy patients were stated for the first time, both in relaxation and contraction.
From the study, a standardised ARFI examination protocol for the masseter can be derived. Future investigators could concentrate only on the superficial component of the muscle, recording 10 valid measurements, during relaxation with ARFI, with respect to the general guidelines of the ARFI examination. We believe that measurements taken while the muscle is at rest could reduce the patient's discomfort and error factors such as muscular fibrillation. The latter statement is based upon the fact that subjects in both groups experience some degree of discomfort during prolonged and repeated muscular contraction. Muscular fibrillation was mostly encountered in Group B patients and this led to inadequate examinations.

\section{Conclusions}

In this preliminary study we used ARFI elastography with SWV for the first time with the purpose of determining the normal and the post-irradiation elasticity of the masseter muscles. The diagnostic value of the method is supported by its low user dependency compared with other methods. More studies are required on large groups of patients to validate the normal values. Radiotherapy induced changes should be further investigated.

Acknowledgements: We hereby express our thanks to the volunteers for their participation in this study.

Conflict of interest: none.

\section{References}

1. Dima R, Ursache M, Forna N. Manducatory muscles electromyographyc evaluation to elderly people. Romanian Journal of Oral Rehabilitation 2008; Pilot number: 59-62.

2. Gavião MBD, Serra-Vicentin MD, Gambareli FR. Correlation between muscle thickness and bite force in children before and after oral rehabilitation-a two year longitudinal study. In: 5th european conference of the international federation for medical and biological engineering. Springer Berlin Heidelberg 2012: 850-853.

3. Kostrzewa-Janicka J, Mierzwinska-Nastalska E, Rolski D, Szczyrek P. Occlusal stabilization splint therapy in orofacial pain and tension-type headache. Adv Exp Med Biol 2013; 788: 181-188.

4. De Rossi SS. Orofacial pain: a primer. Dent Clin North Am 2013; 57: 383-392.

5. Lupsor Platon M, Stefanescu H, Feier D, Maniu A, Badea R. Performance of unidimensional transient elastography in staging chronic hepatitis C. Results from a cohort of 1,202 biopsied patients from one single center. J Gastrointestin Liver Dis 2013; 22: 157-166. 
6. Mueller S, Millonig G, Sarovska L, et al. Increased liver stiffness in alcoholic liver disease: differentiating $\neg$ fibrosis from steatohepatitis. World J Gastroenterol 2010; 16: 966972.

7. Bhatia KS, Lee YY, Yuen EH, Ahuja AT. Ultrasound elastography in the head and neck. Part I. Basic principles and practical aspects. Cancer Imaging 2013; 13: 253-259.

8. Bhatia KS, Lee YY, Yuen EH, Ahuja AT. Ultrasound elastography in the head and neck. Part II. Accuracy for malignancy. Cancer Imaging 2013; 13: 260-276.

9. Ying M, Bhatia KS, Lee YP, Yuen HY, Ahuja AT. Review of ultrasonography of malignant neck nodes: greyscale, Doppler, contrast enhancement and elastography. Cancer Imaging 2014; 13: 658-669.

10. Cakir B, Aydin C, Korukluoglu B, et al. Diagnostic value of elastosonographically determined strain index in the differential diagnosis of benign and malignant thyroid nodules. Endocrine 2011; 39: 89-98.

11. Guazzaroni M, Spinelli A, Coco I, Del Giudice C, Girardi V, Simonetti G. Value of strain-ratio on thyroid real-time sonoelastography. Radiol Med 2014; 119: 149-155.

12. Badea AF, Tamas Szora A, Ciuleanu E, et al. ARFI quantitative elastography of the submandibular glands. Normal measurements and the diagnosis value of the method in radiation submaxillitis. Med Ultrason 2013; 15: 173-179.

13. Westerland O, Howlett D. Sonoelastography techniques in the evaluation and diagnosis of parotid neoplasms. Eur Radiol 2012; 22: 966-969.

14. Bhatia KS, Rasalkar DD, Lee YP, et al. Evaluation of realtime qualitative sonoelastography of focal lesions in the parotid and submandibular glands: applications and limitations. Eur Radiol 2010; 20: 1958-1964.

15. Syversveen T, Midtvedt K, Berstad AE, Brabrand K, Strom EH, Abildgaard A. Tissue elasticity estimated by acoustic radiation force impulse quantification depends on the applied transducer force: an experimental study in kidney transplant patients. Eur Radiol 2012; 22 :2130-2137.

16. Schumacher GH. Funktionelle Morphologie der Kaumuskulatur. Jena: VEB Gustav Fischer Verlag, 1961.

17. van Eijden TM, Turkawski SJ. Morphology and physiology of masticatory muscle motor units. Crit Rev Oral Biol Med 2001; 12: 76-91.

18. Hannam AG, McMillan AS. Internal organization in the human jaw muscles. Crit Rev Oral Biol Med 1994; 5: 55-89.

19. Emshoff R, Bertram S, Strobl H. Ultrasonographic crosssectional characteristics of muscles of the head and neck.
Oral Surg Oral Med Oral Pathol Oral Radiol Endod 1999; 87: 93-106.

20. Serra MD, Duarte Gavião MB, dos Santos Uchôa MN. The use of ultrasound in the investigation of the muscles of mastication. Ultrasound Med Biol 2008; 34: 1875-1884.

21. Chandu A, Suvinen TI, Reade PC, Borromeo GL. Electromyographic activity of frontalis and sternocleidomastoid muscles in patients with temporomandibular disorders. J Oral Rehabil 2005; 32: 571-576.

22. Arda K, Ciledag N, Aktas E, Aribas BK, Kose K. Quantitative assessment of normal soft-tissue elasticity using shearwave ultrasound elastography. AJR Am J Roentgenol 2011; 197: 532-536.

23. Niitsu M, Michizaki A, Endo A, Takei H, Yanagisawa O. Muscle hardness measurement by using ultrasound elastography: a feasibility study. Acta Radiol 2011; 52: 99-105.

24. Sikdar S, Shah JP, Gilliams E, Gebreab T, Gerber LH. Assessment of myofascial trigger points (MTrPs): A new application of ultrasound imaging and vibration sonoelastography. Conf Proc IEEE Eng Med Biol Soc 2008: 55855588.

25. Drakonaki EE, Allen GM. Magnetic resonance imaging, ultrasound and real-time ultrasound elastography of the thigh muscles in congenital muscle dystrophy. Skeletal Radiol 2010; 39: 391-396.

26. Botar-Jid C, Damian L, Dudea SM, Vasilescu D, Rednic S, Badea R. The contribution of ultrasonography and sonoelastography in assessment of myositis. Med Ultrason 2010; 12: $120-126$.

27. Kuo WH, Jian DW, Wang TG, Wang YC. Neck muscle stiffness quantified by sonoelastography is correlated with body mass index and chronic neck pain symptoms. Ultrasound Med Biol 2013; 39: 1356-1361.

28. Ariji Y, Nakayama M, Taguchi A, et al. Intramuscular changes of soft and hard areas after low-level static contraction of the masseter muscle and the correlations with muscle hardness and increase in water content: evaluations with sonographic elastography and magnetic resonance imaging. Oral Surg Oral Med Oral Pathol Oral Radiol 2013; 116: 354-361.

29. Jurdana M. Radiation effects on skeletal muscle. Radiol Oncol 2008;42:15-22.

30. Lefaix JL, Delanian S, Leplat JJ, et al. Radiation induced cutaneo-muscular fibrosis: major therapeutic efficacy of liposomal $\mathrm{Cu} / \mathrm{Zn}$ superoxide dismutase. Bull Cancer 1993; 80: 799-807. 